\section{In vitro window into Rett syndrome}

\section{By Tim Fulmer, Senior Writer}

Researchers at the University of California, San Diego and the Salk Institute for Biological Studies have developed a cell culture-based model of Rett syndrome, an autism-related disorder. ${ }^{1}$ They hope to use the model in combination with animal models to identify compounds to treat the disease, for which there are no marketed drugs.

Rett syndrome is a neurological disorder caused by mutations in the gene that encodes methyl CpG binding protein 2 (MECP2; RTT). The syndrome is characterized by autism-like symptoms that are not detectable until the patient is 6-18 months of age. When they do appear, the symptoms are severe, with noticeable regression of language and motor skills.

The year or longer delay and sudden onset of severe symptoms have led some researchers to propose that impairment of neuronal growth and function may occur during the disease's presymptomatic stages, providing a rationale for blocking those impairments. ${ }^{2-4}$

Thus, a team of researchers led by Alysson Muotri and Fred Gage set out to identify the neuronal processes that underlie the development of Rett syndrome. The group first designed a cell culture-based model. By studying the in vitro properties of neurons from Rett patients, the researchers hoped to get a better handle on which cellular pathways to target.

Muotri is an assistant professor of medicine at UCSD. Gage is a professor of genetics at the Salk Institute.

The researchers isolated fibroblasts from Rett patients and reprogrammed the skin cells to become induced pluripotent stem (iPS) cells. The iPS cells then were differentiated into neurons that could serve as cell culture models of the syndrome.

In vitro, the iPS cell-derived neurons had the same impaired growth characteristics as neurons isolated from Rett syndrome patients. These included noticeable morphological aberrations, disturbances in calcium homeostasis and fewer synapses compared with those in neurons from healthy controls.

Finally, the researchers looked at whether any of the aberrations of the cultured Rett neurons could be reversed therapeutically. To do that, they treated the cells with insulin-like growth factor-1 (IGF-1), which is known to reverse some Rett syndrome symptoms in mice. ${ }^{5}$

Indeed, IGF-1 increased the number of synapses compared with vehicle control, suggesting that the growth factor could correct at least some of the deficiencies of the cultured Rett neurons.

In conclusion, the authors wrote that the iPS cell-derived Rett syndrome model "not only can recapitulate some aspects of a genetic disease but also can be used to better design and anticipate results from translational medicine."

The authors added that the model "has the potential to lead to the discovery of new compounds to treat Rett syndrome and other forms of autism spectrum disorder."

The findings were published in Cell.

Corresponding author Muotri told SciBX that he now hopes to use the iPS cell-derived neurons as the basis of a high throughput screening platform to discover small molecules to treat Rett syndrome and other autism-related disorders.

"We believe such a screen could be used in combination with animal models of neuropsychiatric disease to identify potential therapeutic candidates," Muotri said. "We'll be looking at a variety of cellular readouts to help us identify the best therapeutic candidates for further testing in animal models. Those readouts will include neuronal morphology and synaptic connectivity, which are often disrupted in neuropsychiatric and autism-related diseases."

Muotri thinks the model described in Cell could be considered a prototype for other cell culture models of neuropsychiatric disease and hopes to collaborate with industry on such studies. "We believe it could be possible to generate similar cell culture models of other forms of autism and neurodegenerative disorders where the fundamental genetic or mutational driver of the disease is known," he said.

The findings are covered by a provisional patent and are available for licensing.

Fulmer, T. SciBX 3(47); doi:10.1038/scibx.2010.1403

Published online Dec. 9, 2010

\section{REFERENCES}

1. Marchetto, M.C.N. et al. Cell; published online Nov. 12, 2010; doi:10.1016/j.cell.2010.10.016

Contact: Alysson R. Muotri, University of California, San Diego,

La Jolla, Calif.

e-mail: muotri@ucsd.edu

2. Charman, T. et al. Brain Dev. 24, 281-283 (2002)

3. De Filippis, B. et al. Genes Brain Behav. 9, 213-223 (2009)

4. Santos, M. et al. Genes Brain Behav. 6, 277-286 (2007)

5. Tropea, D. et al. Proc. Natl. Acad. Sci. USA 106, 2029-2034 (2009)

COMPANIES AND INSTITUTIONS MENTIONED

Salk Institute for Biological Studies, La Jolla, Calif.

University of California, San Diego, La Jolla, Calif. 